\title{
Approximate Analytical Solutions of the Pseudospin Symmetric Dirac Equation for Exponential-type Potentials
}

\author{
Altuğ Arda, ${ }^{1, *}$ Ramazan Sever, ${ }^{2, \dagger}$ and Cevdet Tezcan ${ }^{3,+}$ \\ ${ }^{1}$ Department of Physics Education, Hacettepe University, 06800, Ankara,Turkey \\ ${ }^{2}$ Department of Physics, Middle East Technical University, 06800, Ankara,Turkey \\ ${ }^{3}$ Faculty of Engineering, Başkent University, Baglıca Campus, Ankara,Turkey
}

(Dated: October 30, 2018)

\begin{abstract}
The solvability of The Dirac equation is studied for the exponential-type potentials with the pseudospin symmetry by using the parametric generalization of the Nikiforov-Uvarov method. The energy eigenvalue equation, and the corresponding Dirac spinors for Morse, Hulthen, and $q$ deformed Rosen-Morse potentials are obtained within the framework of an approximation to the spin-orbit coupling term, so the solutions are given for any value of the spin-orbit quantum number $\kappa=0$, or $\kappa \neq 0$.
\end{abstract}

Keywords: Pseudospin symmetry, Morse potential, Hulthen potential, q-deformed Rosen-Morse potential, Dirac equation, Nikiforov-Uvarov Method

PACS numbers: 03.65.-w; 03.65.Ge; 12.39.Fd

${ }^{*}$ E-mail: arda@hacettepe.edu.tr
${ }^{\dagger}$ E-mail: sever@metu.edu.tr
${ }^{\ddagger}$ E-mail: ctezcan@baskent.edu.tr 


\section{INTRODUCTION}

The concept of pseudo-spin was constructed firstly in spherical nuclei $[1,2]$, and it is observed experimentally that the single particle levels labeled as pseudo-spin doublets are very close in energy [3]. The pseudo-spin doublets in nuclei are decomposed by using radial $\left(n_{r}\right)$, orbital $(\ell)$, and total angular momentum $(j)$ quantum numbers as $\left(n_{r}, \ell, j=\ell+1 / 2\right)$, and $\left(n_{r}-1, \ell+2, j=\ell+3 / 2\right)$. The pseudo orbital angular momentum, $\tilde{\ell}=\ell+1$, and the pseudo spin, $\widetilde{s}$, quantum numbers give total angular momentum, $j=\widetilde{\ell}+\widetilde{s}$, and the pseudo-spin doublets are degenerate with respect to pseudo spin, $\widetilde{s}$ [4]. The pseudo-spin doublets occur in nuclei, when the magnitude of scalar, $V_{s}(r)$, and vector, $V_{v}(r)$, potentials are nearly equal, with opposite $\operatorname{sign}$, i.e., $V_{s}(r) \simeq-V_{v}(r)$ [5]. The pseudo-spin symmetry is studied based on Dirac equation in real nuclei, and shown that it is related with the competition between the centrifugal barrier, and pseudo-spin orbital potential [6]. The pseudo-spin concept is discussed in deformed nuclei [7], and exotic nuclei as well [3]. It is observed that the pseudo-spin symmetry is also an important one in the case of triaxiality $[8]$.

The pseudo-spin orbital potential creates the splitting of the pseudo-spin doublets, and the pseudo-spin symmetry is exact symmetry in real nuclei, when the derivative of the difference between scalar, and vector potentials vanishes, but the above condition gives a good symmetry for exotic nuclei $[3,6]$. The pseudo-spin symmetry is identified as a $S U(2)$ symmetry of the Dirac Hamiltonian, under the condition that the sum of scalar, and vector potentials is equal to zero [9]. Recently, it is pointed out that the shape of the lower components of the Dirac spinor for the doublets is the same, when the pseudo-spin doublets are degenerate [4]. It is showed that it becomes possible to construct a map which relates the normal state $(\ell, s)$ with the pseudo state $(\widetilde{\ell}, \widetilde{s})$ by applying of the helicity operator to the non-relativistic single-particle eigenfunction to understand of the mechanism to generate the pseudo-spin symmetry [10]. The pseudo-spin concept is discussed in the non-relativistic harmonic oscillator, and obtained that the condition between the coefficients of spin-orbit, and orbit-orbit terms in the case of non-relativistic single-particle Hamiltonian having the pseudo-spin symmetry is consistent with the result obtained relativistic mean-field theory $[11,12]$.

The pseudo-spin symmetry concept has found a great application area, especially in 
nuclear theory. The identical bands observed in nuclei are explained by using the pseudospin symmetry [13]. The idea of the pseudo-spin has been considered to be useful to construct an effective shell-model coupling scheme [14]. Some features of nuclei, such as deformation, and superdeformation can be explained in the context of the pseudo-spin symmetry [13, 15, 16].

Recently, the solutions of the Klein-Gordon, and Dirac equations including the spin-orbit coupling term have been studied by many authors for different potentials, such as Morse potential [17-20], Pöschl-Teller potential [21-23], Woods-Saxon potential [24], Eckart [25-28], harmonic oscillator [29, 30], three parameter diatomic molecular potential [31], and angledependent potential [32]. In the present work, we deal with the approximate solutions, and corresponding wave functions of the Dirac equation including spin-orbit coupling term under the exact pseudo-spin symmetry for Morse, Hulthén, and q-deformed Rosen-Morse potentials. We point out that the parametric generalization of the Nikiforov-Uvarov method can be applied to the Dirac equation with the above potentials, and the energy eigenvalue equation, and corresponding eigenfunctions can be obtained for the values of spin-orbit quantum number $\kappa=0$, or $\kappa \neq 0$.

\section{THE DIRAC EQUATION WITH SPIN-ORBIT COUPLING}

The Dirac equation for a fermion with mass $m$ moving in an external scalar, and vector potentials reads $(\hbar=c=1)$

$$
\left[\alpha \cdot \hat{p}+\beta\left[m+V_{s}(r)\right]+V_{v}(r)\right] \Psi(r)=E \Psi(r)
$$

where $E$ is the energy of the particle, $\hat{p}$ is the three-momentum operator, and $\alpha$, and $\beta$ are the $4 \times 4$ Dirac matrices written in terms of $2 \times 2$ Pauli matrices, and unit matrix. Under the consideration that the system has a spherical symmetry for which the potential fields depend on the radial coordinate, the quantum state of the particle is labeled by the quantum number set $\left(n_{r}, j, m, \kappa\right)$, where $m$ is the projection of the total angular momentum on the $z$-axis, and $\kappa= \pm(j+1 / 2)$ is the eigenvalues of the operator $\hat{\kappa}=-\beta(\hat{\sigma} \cdot \hat{L}+1)[33]$. Here, $\kappa=-(j+1 / 2)$ denotes the aligned $\operatorname{spin}\left(s_{1 / 2}, p_{3 / 2}\right.$, etc. $)$, and $\kappa=+(j+1 / 2)$ denotes the

unaligned spin $\left(p_{1 / 2}, d_{3 / 2}\right.$, etc. $)$. The spherically symmetric Dirac wave function can than be 
written in terms of upper, and lower components as [34]

$$
\Psi(r)=\frac{1}{r}\left(\begin{array}{c}
f(r)\left[Y_{\ell} \chi\right]_{m}^{j} \\
i g(r)\left[Y_{\tilde{\ell}} \chi\right]_{m}^{j}
\end{array}\right)
$$

where $f(r)$, and $g(r)$ are the radial wave functions, $Y_{\ell}(\theta, \phi)$, and $\chi$ are the spherical, and spin functions, respectively. Substituting Eq (2) into Eq. (1), we get the following radial Dirac equations

$$
\begin{aligned}
& \left(\frac{d}{d r}+\frac{\kappa}{r}\right) f(r)-[M(r)+\epsilon] g(r)=0, \\
& \left(\frac{d}{d r}-\frac{\kappa}{r}\right) g(r)-[M(r)-\epsilon] f(r)=0 .
\end{aligned}
$$

where $M(r)=m+V_{s}(r)$, and $\epsilon=E-V_{v}(r)$. Using the expression for $g(r)$ obtained from Eq. (3), and inserting it into Eq. (4), we have two second order differential equations including spin-orbit coupling term

$$
\begin{aligned}
& \left\{\frac{d^{2}}{d r^{2}}-\frac{\kappa(\kappa+1)}{r^{2}}-\left[M^{2}(r)-\epsilon^{2}\right]\right\} f(r)=\left\{\frac{1}{M(r)+\epsilon} \frac{d}{d r}\left[V_{s}(r)-V_{v}(r)\right]\left(\frac{d}{d r}+\frac{\kappa}{r}\right)\right\} f(r), \\
& \left\{\frac{d^{2}}{d r^{2}}-\frac{\kappa(\kappa-1)}{r^{2}}-\left[M^{2}(r)-\epsilon^{2}\right]\right\} g(r)=\left\{\frac{1}{M(r)-\epsilon} \frac{d}{d r}\left[V_{s}(r)+V_{v}(r)\right]\left(\frac{d}{d r}-\frac{\kappa}{r}\right)\right\} g(r),
\end{aligned}
$$

Under the condition of the exact pseudo-spin symmetry, i.e., $\frac{d}{d r}\left[V_{v}(r)+V_{s}(r)\right]=0$, or $V_{v}(r)+V_{s}(r)=C=$ const., Eq. (6) gives

$$
\left\{\frac{d^{2}}{d r^{2}}-\frac{\kappa(\kappa-1)}{r^{2}}+[m-E+C]\left[V_{v}(r)-V_{s}(r)\right]\right\} g(r)=\left[m^{2}-E^{2}+C(m+E)\right] g(r)
$$

From the last equation, the energy eigenvalues depend also on the quantum number $\tilde{\ell}$ because of the relations given by $\kappa(\kappa-1)=\tilde{\ell}(\tilde{\ell}+1)$, and $\kappa(\kappa+1)=\ell(\ell+1)$. So, the energy eigenstates with $j=\tilde{\ell} \pm 1 / 2$ are degenerate for $\tilde{\ell} \neq 0$, which gives the situation of the exact pseudo-spin symmetry in the Dirac equation.

In the present work, we intend to solve the last equation four different potentials, Morse, Hulthén, and q-deformed Rosen-Morse potentials, namely. The Dirac equation in Eq. (7) can not be solved exactly because of the spin-orbit coupling term. So, we use the Pekeris 
approximation [35] to find the suitable expression instead of the spin-orbit coupling term in the case of Morse potential. This approximation makes possible to write the interaction term

in the form of the Morse potential in terms of new parameters $D_{i}(i=0,1,2)$. In the case of Hulthén, and q-deformed Rosen-Morse potential, we introduce the following approximation instead of the spin-orbit coupling term [22]

$$
\frac{1}{r^{2}} \approx \frac{\alpha^{2} e^{-\alpha r}}{\left(1-e^{-\alpha r}\right)^{2}}
$$

which gives a second order differential equation from Eq. (7) without $\left(1 / r^{2}\right)$-term. In all case of potentials, we use the parametric generalization of the Nikiforov-Uvarov method [36], and so we prove that the parametric version of the method can be applied to the Dirac equation with Morse, Pöschl-Teller, Hulthén, and q-deformed Rosen-Morse potential.

\section{NIKIFOROV-UVAROV METHOD}

By using an appropriate coordinate transformation, the Schrödinger equation is transformed into the following form

$$
\Psi^{\prime \prime}(s)+\frac{\tilde{\tau}(s)}{\sigma(s)} \Psi^{\prime}(s)+\frac{\tilde{\sigma}(s)}{\sigma^{2}(s)} \Psi(s)=0
$$

where $\sigma(s), \tilde{\sigma}(s)$ are polynomials, at most second degree, and $\tilde{\tau}(s)$ is a first degree polynomial. In the NU-method, the polynomial $\pi(s)$, and the parameter $k$ are required, and defined as

$$
\pi(s)=\frac{1}{2}\left[\sigma^{\prime}(s)-\tilde{\tau}(s)\right] \pm \sqrt{\frac{1}{4}\left[\sigma^{\prime}(s)-\tilde{\tau}(s)\right]^{2}-\tilde{\sigma}(s)+k \sigma(s)}
$$

and

$$
\lambda=k+\pi^{\prime}(s)
$$

where $\lambda$ is a constant. The function under the square root in the polynomial in $\pi(s)$ in Eq. (10) must be square of a polynomial in order that $\pi(s)$ be a first degree polynomial, so the 
derivative of $\pi(s)$ is a constant, and this defines the constant $k$. Replacing $k$ into Eq. (10), we define

$$
\tau(s)=\tilde{\tau}(s)+2 \pi(s)
$$

where the derivative of $\tau(s)$ should be negative [35], which let us know the choice of the solution. The hypergeometric type equation in Eq. (9) has a particular solution with degree $n$, if $\lambda$ in Eq. (11) satisfies

$$
\lambda=\lambda_{n}=-n \tau^{\prime}-\frac{\left[n(n-1) \sigma^{\prime \prime}\right]}{2}, \quad n=0,1,2, \ldots
$$

To obtain the solution of Eq. (9) it is assumed that the solution is a product of two independent parts

$$
\Psi(s)=\phi(s) y(s)
$$

where $y(s)$ can be written as

$$
y_{n}(s)=\frac{a_{n}}{\rho(s)} \frac{d^{n}}{d s^{n}}\left[\sigma^{n}(s) \rho(s)\right],
$$

where $a_{n}$ is normalization constant, and the function $\rho(s)$ is the weight function, and should satisfy the condition

$$
\frac{d \sigma(s)}{d s} \rho(s)+\sigma(s) \frac{d \rho(s)}{d s}=\tau(s) \rho(s),
$$

The other factor is defined as

$$
\frac{\phi^{\prime}(s)}{\phi(s)}=\frac{\pi(s)}{\sigma(s)}
$$

In order to clarify the parametric generalization of the NU method, let us take the following equation, which represents a general form of the Schrödinger-like equation written for any potential, 


$$
\left[\frac{d^{2}}{d s^{2}}+\frac{\alpha_{1}-\alpha_{2} s}{s\left(1-\alpha_{3} s\right)} \frac{d}{d s}+\frac{-\xi_{1} s^{2}+\xi_{2} s-\xi_{3}}{\left[s\left(1-\alpha_{3} s\right)\right]^{2}}\right] \Psi(s)=0 .
$$

When Eq. (18) is compared with Eq. (9), we get

$$
\tilde{\tau}(s)=\alpha_{1}-\alpha_{2} s ; \sigma(s)=s\left(1-\alpha_{3} s\right) ; \tilde{\sigma}(s)=-\xi_{1} s^{2}+\xi_{2} s-\xi_{3} .
$$

Substituting these into Eq. (10)

$$
\pi(s)=\alpha_{4}+\alpha_{5} s \pm \sqrt{\left(\alpha_{6}-k \alpha_{3}\right) s^{2}+\left(\alpha_{7}+k\right) s+\alpha_{8}}
$$

where the parameter set are

$$
\begin{aligned}
& \alpha_{4}=\frac{1}{2}\left(1-\alpha_{1}\right), \alpha_{5}=\frac{1}{2}\left(\alpha_{2}-2 \alpha_{3}\right), \\
& \alpha_{6}=\alpha_{5}^{2}+\xi_{1}, \quad \alpha_{7}=2 \alpha_{4} \alpha_{5}-\xi_{2}, \\
& \alpha_{8}=\alpha_{4}^{2}+\xi_{3} .
\end{aligned}
$$

In NU-method, the function under the square root in Eq. (20) must be the square of a polynomial [35]. This condition gives the roots of the parameter $k$, and they can be written as

$$
k_{1,2}=-\left(\alpha_{7}+2 \alpha_{3} \alpha_{8}\right) \pm 2 \sqrt{\alpha_{8} \alpha_{9}}
$$

where the $k$-values can be real or imaginary, and $\alpha_{9}=\alpha_{3} \alpha_{7}+\alpha_{3}^{2} \alpha_{8}+\alpha_{6}$. Different $k$ 's lead to the different $\pi(s)$ 's. For

$$
k=-\left(\alpha_{7}+2 \alpha_{3} \alpha_{8}\right)-2 \sqrt{\alpha_{8} \alpha_{9}}
$$

$\pi(s)$ becomes

$$
\pi(s)=\alpha_{4}+\alpha_{5} s-\left[\left(\sqrt{\alpha_{9}}+\alpha_{3} \sqrt{\alpha_{8}}\right) s-\sqrt{\alpha_{8}}\right],
$$


and also

$$
\tau(s)=\alpha_{1}+2 \alpha_{4}-\left(\alpha_{2}-2 \alpha_{5}\right) s-2\left[\left(\sqrt{\alpha_{9}}+\alpha_{3} \sqrt{\alpha_{8}}\right) s-\sqrt{\alpha_{8}}\right]
$$

Thus, we impose the following for satisfying the condition that the derivative of the function $\tau(s)$ should be negative in the method

$$
\begin{aligned}
\tau^{\prime}(s) & =-\left(\alpha_{2}-2 \alpha_{5}\right)-2\left(\sqrt{\alpha_{9}}+\alpha_{3} \sqrt{\alpha_{8}}\right) \\
& =-2 \alpha_{3}-2\left(\sqrt{\alpha_{9}}+\alpha_{3} \sqrt{\alpha_{8}}\right) \quad<0 .
\end{aligned}
$$

From Eqs. (11), (12), (25), and (26), and equating Eq. (11) with the condition that $\lambda$ should satisfy given by Eq. (13), we find the eigenvalue equation

$$
\begin{aligned}
\alpha_{2} n-(2 n+1) \alpha_{5} & +(2 n+1)\left(\sqrt{\alpha_{9}}+\alpha_{3} \sqrt{\alpha_{8}}\right)+n(n-1) \alpha_{3} \\
& +\alpha_{7}+2 \alpha_{3} \alpha_{8}+2 \sqrt{\alpha_{8} \alpha_{9}}=0 .
\end{aligned}
$$

We get from Eq. (16)

$$
\rho(s)=s^{\alpha_{10}-1}\left(1-\alpha_{3} s\right)^{\frac{\alpha_{11}}{\alpha_{3}}-\alpha_{10}-1}
$$

and substituting into Eq. (15) gives

$$
y_{n}(s)=P_{n}^{\left(\alpha_{10}-1, \frac{\alpha_{11}}{\alpha_{3}}-\alpha_{10}-1\right)}\left(1-2 \alpha_{3} s\right)
$$

where

$$
\alpha_{10}=\alpha_{1}+2 \alpha_{4}+2 \sqrt{\alpha_{8}} ; \alpha_{11}=\alpha_{2}-2 \alpha_{5}+2\left(\sqrt{\alpha_{9}}+\alpha_{3} \sqrt{\alpha_{8}}\right)
$$

and $P_{n}^{(\alpha, \beta)}\left(1-2 \alpha_{3} s\right)$ are the Jacobi polynomials. From Eq. (17), one gets

$$
\phi(s)=s^{\alpha_{12}}\left(1-\alpha_{3} s\right)^{-\alpha_{12}-\frac{\alpha_{13}}{\alpha_{3}}}
$$


then the general solution $\Psi(s)=\phi(s) y(s)$ becomes

$$
\Psi(s)=s^{\alpha_{12}}\left(1-\alpha_{3} s\right)^{-\alpha_{12}-\frac{\alpha_{13}}{\alpha_{3}}} P_{n}^{\left(\alpha_{10}-1, \frac{\alpha_{11}}{\alpha_{3}}-\alpha_{10}-1\right)}\left(1-2 \alpha_{3} s\right)
$$

where

$$
\alpha_{12}=\alpha_{4}+\sqrt{\alpha_{8}} ; \alpha_{13}=\alpha_{5}-\left(\sqrt{\alpha_{9}}+\alpha_{3} \sqrt{\alpha_{8}}\right) \text {. }
$$

\section{BOUND-STATE SOLUTIONS}

\section{A. Morse Potential}

We set the difference between scalar, and vector potentials in Eq. (6) as the Morse potential [37] given by

$$
V_{\text {Morse }}(r)=D\left[e^{-2 \alpha x}-2 e^{-\alpha x}\right]
$$

where $D$ is the dissociation energy of the potential, $\alpha=a r_{0}, x=\frac{r}{r_{0}}-1, r_{0}$ is the equilibrium distance, and the positive parameter $a$ is the potential width. According to the Pekeris approximation, the spin-orbit coupling term can be written in terms of three new parameters $D_{0}, D_{1}$, and $D_{2}$ as

$$
\frac{\kappa(\kappa-1)}{r^{2}} \simeq \gamma\left(D_{0}+D_{1} e^{-\alpha x}+D_{2} e^{-2 \alpha x}\right)
$$

where $\gamma=\kappa(\kappa-1)) r_{0}^{2}$ (see Ref [17], for details). Substituting Eq. (34), and (35) into Eq. (6), we get

$$
\frac{d^{2} g(x)}{d x^{2}}+\left\{a_{3}^{2} e^{-2 \alpha x}-a_{2}^{2} e^{-\alpha x}-a_{1}^{2}\right\} g(x)=0
$$

where $a_{1}^{2}=r_{0}^{2}\left[\gamma D_{0}+\Sigma_{m}(m+E)\right], a_{2}^{2}=r_{0}^{2}\left(\gamma D_{1}+2 D \Sigma_{m}\right)$, and $a_{3}^{2}=r_{0}^{2}\left(D \Sigma_{m}-\gamma D_{2}\right)$ in the above equations. By using the new variable $s=e^{-\alpha x}$, we obtain 


$$
\frac{d^{2} g(s)}{d s^{2}}+\frac{1}{s} \frac{d g(s)}{d s}+\left\{a_{3}^{2} \beta^{2}-\frac{a_{2}^{2} \beta^{2}}{s}-\frac{a_{1}^{2} \beta^{2}}{s^{2}}\right\} g(s)=0
$$

where $\beta^{2}=1 / \alpha^{2}$, and $\Sigma_{m}=m-E+C$. Comparing the Eq. (37) with Eq. (18), we obtain the following parameter set

$$
\begin{array}{ll}
\alpha_{1}=1, & -\xi_{1}=a_{3}^{2} \beta^{2} \\
\alpha_{2}=0, & \xi_{2}=-a_{2}^{2} \beta^{2} \\
\alpha_{3}=0, & -\xi_{3}=-a_{1}^{2} \beta^{2} \\
\alpha_{4}=0, & \alpha_{5}=0 \\
\alpha_{6}=\xi_{1}, & \alpha_{7}=-\xi_{2} \\
\alpha_{8}=\xi_{3}, & \alpha_{9}=\xi_{1} \\
\alpha_{10}=1+2 \sqrt{\xi_{3}}, & \alpha_{11}=2 \sqrt{\xi_{1}} \\
\alpha_{12}=\sqrt{\xi_{3}}, & \alpha_{13}=-\sqrt{\xi_{1}}
\end{array}
$$

Substituting the above expressions in Eq. (27), we obtain the energy eigenvalue equation of a fermion moving in the Morse potential under the exact pseudo-spin symmetry

$$
\begin{aligned}
(2 n+1) \sqrt{\gamma D_{2}-D \Sigma_{m}} & +\beta r_{0}\left(\gamma D_{1}+2 D \Sigma_{m}\right) \\
& +2 \beta r_{0} \sqrt{\left[\gamma D_{0}+\Sigma_{m}(m+E)\right]\left(\gamma D_{2}-D \Sigma_{m}\right)}=0
\end{aligned}
$$

and the lower spinor component from Eq. (32)

$$
\begin{aligned}
g(s) & =s^{\beta r_{0} \sqrt{\gamma D_{0}+\Sigma_{m}(m+E)}} e^{-\beta r_{0} \sqrt{\gamma D_{2}-D \Sigma_{m}} s} \\
& \times L_{n}^{2 \beta r_{0} \sqrt{\gamma D_{0}+\Sigma_{m}(m+E)}}\left(2 \beta r_{0} \sqrt{\gamma D_{2}-D \Sigma_{m}} s\right),
\end{aligned}
$$

where $L_{n}^{(k)}(x)$ are the Laguerre polynomials.

We give the upper spinor component from Eq. (4), by using Eq. (40)

$$
\begin{aligned}
f(s) & =a \Sigma_{m}^{-1} s^{\beta r_{0} \sqrt{\gamma D_{0}+\Sigma_{m}(m+E)}} e^{-\beta r_{0} \sqrt{\gamma D_{2}-D \Sigma_{m}} s} \\
& \times\left\{\beta r _ { 0 } \sqrt { \gamma D _ { 2 } - D \Sigma _ { m } } s \left[L_{n}^{2 \beta r_{0} \sqrt{\gamma D_{0}+\Sigma_{m}(m+E)}}\left(2 \beta r_{0} \sqrt{\gamma D_{2}-D \Sigma_{m}} s\right)\right.\right.
\end{aligned}
$$




$$
\begin{aligned}
& \left.+L_{n-1}^{1+2 \beta r_{0} \sqrt{\gamma D_{0}+\Sigma_{m}(m+E)}}\left(2 \beta r_{0} \sqrt{\gamma D_{2}-D \Sigma_{m}} s\right)\right] \\
& -\left[\beta r_{0} \sqrt{\gamma D_{0}+\Sigma_{m}(m+E)}+\frac{\kappa}{a r_{0}-\ln s}\right] \\
& \left.\times L_{n}^{2 \beta r_{0} \sqrt{\gamma D_{0}+\Sigma_{m}(m+E)}}\left(2 \beta r_{0} \sqrt{\gamma D_{2}-D \Sigma_{m}} s\right)\right\} \text {. }
\end{aligned}
$$

where we use some recursion relations of the Laguerre polynomials.

\section{B. Hulthén Potential}

The Hulthén potential reads

$$
V(r)=-V_{0} \frac{e^{-x}}{1-e^{-x}}
$$

where $x=\frac{r}{r_{0}}$, and $r_{0}$ denotes the spatial range $[38,39]$. We choose the difference between scalar, and vector potentials as Hulthén potential potential, and using the approximation in Eq. (8), which becomes $\frac{1}{r^{2}} \simeq \frac{e^{x}}{r_{0}^{2}\left(e^{x}-1\right)^{2}}$ in the present case, we obtain from Eq. (6)

$$
\frac{d^{2} g(r)}{d r^{2}}-\left\{\frac{\kappa(\kappa-1) e^{x}}{r_{0}^{2}\left(e^{x}-1\right)^{2}}+\Sigma_{m}\left(m+E+\frac{V_{0}}{e^{x}-1}\right)\right\} g(r)=0,
$$

Using the new variable $s=e^{-x}$, Eq. (43) becomes

$$
\begin{aligned}
\frac{d^{2} g(s)}{d s^{2}} & +\frac{1-s}{s(1-s)} \frac{d g(s)}{d s}+\frac{1}{[s(1-s)]^{2}}\left\{-r_{0}^{2} \Sigma_{m}(m+E)\right. \\
& +\left[-\kappa(\kappa-1)+2 r_{0}^{2} \Sigma_{m}(m+E)-r_{0}^{2} \Sigma_{m} V_{0}\right] s \\
& \left.+\left[-r_{0}^{2} \Sigma_{m}(m+E)+r_{0}^{2} \Sigma_{m} V_{0}\right] s^{2}\right\} g(s)=0
\end{aligned}
$$

Comparing with Eq. (18), we get 


$$
\begin{array}{ll}
\alpha_{1}=1, & -\xi_{1}=r_{0}^{2} \Sigma_{m}\left[-m-E+V_{0}\right] \\
\alpha_{2}=1, & \xi_{2}=r_{0}^{2} \Sigma_{m}\left[2(m+E)-V_{0}\right]-\kappa(\kappa-1) \\
\alpha_{3}=1, & -\xi_{3}=-r_{0}^{2} \Sigma_{m}(m+E) \\
\alpha_{4}=0, & \alpha_{5}=-1 / 2 \\
\alpha_{6}=\frac{1}{4}+\xi_{1}, & \alpha_{7}=-\xi_{2} \\
\alpha_{8}=\xi_{3}, & \alpha_{9}=\xi_{1}-\xi_{2}+\xi_{3}+\frac{1}{4} \\
\alpha_{10}=1+2 \sqrt{\xi_{3}}, & \alpha_{11}=2+2\left(\sqrt{\xi_{1}-\xi_{2}+\xi_{3}+\frac{1}{4}}+\sqrt{\xi_{3}}\right) \\
\alpha_{12}=\sqrt{\xi_{3}}, & \alpha_{13}=-\frac{1}{2}-\sqrt{\xi_{1}-\xi_{2}+\xi_{3}+\frac{1}{4}}-\sqrt{\xi_{3}}
\end{array}
$$

The energy eigenvalue equation is written from Eq. (27)

$$
\begin{array}{r}
r_{0} \sqrt{\Sigma_{m}(m+E)}[2 n+1+\sqrt{4 \kappa(\kappa-1)+1}]+\left(n+\frac{1}{2}\right) \sqrt{4 \kappa(\kappa-1)+1} \\
+n(n+1)+\kappa(\kappa-1)+r_{0}^{2} \Sigma_{m} V_{0}+\frac{1}{2}=0
\end{array}
$$

and corresponding lower, and upper Dirac spinors by using Eqs. (4) and (32), respectively,

$$
\begin{aligned}
& g(s)=s^{r_{0} \sqrt{\Sigma_{m}(m+E)}}(1-s)^{\frac{1}{2}[1+\sqrt{4 \kappa(\kappa-1)+1}]} \\
& \times P_{n}^{\left(2 r_{0} \sqrt{\Sigma_{m}(m+E)}, \sqrt{4 \kappa(\kappa-1)+1}\right)}(1-2 s), \\
& f(s)=\left(r_{0} \Sigma_{m}\right)^{-1} s^{r_{0} \sqrt{\Sigma_{m}(m+E)}}(1-s)^{\frac{1}{2}[1+\sqrt{4 \kappa(\kappa-1)+1}]} \\
& \times\left\{\left[-r_{0} \sqrt{\Sigma_{m}(m+E)}+\frac{1}{2}[1+\sqrt{4 \kappa(\kappa-1)+1}] \frac{s}{1-s}\right.\right. \\
& \left.+\frac{\kappa}{l n s}\right] P_{n}^{\left(2 r_{0} \sqrt{\Sigma_{m}(m+E)}, \sqrt{4 \kappa(\kappa-1)+1}\right)}(1-2 s) \\
& +\left(n+2 r_{0} \sqrt{\Sigma_{m}(m+E)}+\sqrt{4 \kappa(\kappa-1)+1}+1\right) s \\
& \times P_{n-1}^{\left(1+2 r_{0} \sqrt{\Sigma_{m}(m+E)}, 1+\sqrt{\kappa(\kappa-1)+1}\right)}(1-2 s) \text {. }
\end{aligned}
$$

where $P_{n}^{(k, m)}(x)$ are the Jacobi polynomials, and we have used some recursion relations of the Jacobi polynomials to obtain Eq. (48).

\section{C. $q$-Deformed Rosen-Morse Potential}

The $q$-deformed Rosen-Morse Potential reads [40] 


$$
V(r)=\frac{V_{1}}{1+q e^{-2 x}}-V_{2} q \frac{e^{-2 x}}{\left(1+q e^{-2 x}\right)^{2}},
$$

where $x=\alpha r$, and $q$ is the deformation parameter.

We prefer to solve the Dirac equation for $\kappa=0$ in Eq. (6) with the PT-symmetric version of the potential by setting to the difference between scalar, and vector potentials

$$
V^{P T}(r)=\frac{V_{1}}{1+q e^{-2 i x}}-V_{2} q \frac{e^{-2 i x}}{\left(1+q e^{-2 i x}\right)^{2}} .
$$

We obtain the following equation under the above consideration by using the transformation $s=-e^{-2 i x}$

$$
\begin{aligned}
\frac{d^{2} g(s)}{d s^{2}} & +\frac{1-q s}{s(1-q s)} \frac{d g(s)}{d s}+\frac{1}{[s(1-q s)]^{2}}\left\{\delta^{2}\left[V_{1} \Sigma_{m}-\Sigma_{m}(m+E)\right]\right. \\
& \left.+\delta^{2} \Sigma_{m}\left[2 q(m+E)-q V_{1}+V_{2}\right] s-\delta^{2} q^{2} \Sigma_{m}(m+E) s^{2}\right\} g(s)=0
\end{aligned}
$$

Comparing the Eq. (51) with Eq. (18), we get

$$
\begin{array}{ll}
\alpha_{1}=1, & -\xi_{1}=\delta^{2} q^{2} \Sigma_{m}(m+E) \\
\alpha_{2}=q, & \xi_{2}=\delta^{2} \Sigma_{m}\left[2 q(m+E)-q V_{1}+V_{2}\right] \\
\alpha_{3}=q, & -\xi_{3}=\delta^{2}\left[V_{1} \Sigma_{m}-\Sigma_{m}(m+E)\right] \\
\alpha_{4}=0, & \alpha_{5}=-q / 2 \\
\alpha_{6}=\frac{q^{2}}{4}+\xi_{1}, & \alpha_{7}=-\xi_{2} \\
\alpha_{8}=\xi_{3}, & \alpha_{9}=\xi_{1}-q \xi_{2}+q^{2} \xi_{3}+\frac{q^{2}}{4} \\
\alpha_{10}=1+2 \sqrt{\xi_{3}}, & \alpha_{11}=2 q+2\left(\sqrt{\xi_{1}-q \xi_{2}+q^{2} \xi_{3}+\frac{2}{4}}+q \sqrt{\xi_{3}}\right) \\
\alpha_{12}=\sqrt{\xi_{3}}, & \alpha_{13}=-\frac{q}{2}-\sqrt{\xi_{1}-q \xi_{2}+q^{1} \xi_{3}+\frac{q^{2}}{4}}+q \sqrt{\xi_{3}}
\end{array}
$$

The energy eigenvalue equation is written from Eq. (27)

$$
\begin{array}{r}
2 \delta \sqrt{\Sigma_{m}\left(m+E-V_{1}\right)}\left[2 n+1+\frac{1}{2} \sqrt{1-\left(4 V_{2} \Sigma_{m}\right) / q}\right]+\left(n+\frac{1}{2}\right) \sqrt{1-\left(4 V_{2} \Sigma_{m}\right) / q} \\
+n(n+1)+\frac{1}{2}-\delta^{2} \Sigma_{m}\left(V_{1}+V_{2}\right) / q=0 .
\end{array}
$$

and corresponding lower, and upper Dirac spinors by using Eqs. (4) and (32), respectively, 


$$
\begin{aligned}
& g(s)=s^{\delta \sqrt{\Sigma_{m}\left(m+E-V_{1}\right)}}(1-q s)^{\frac{1}{2}\left[1+\sqrt{1-\left(4 V_{2} \Sigma_{m}\right) / q}\right]} \\
& \times P_{n}^{\left(2 \delta \sqrt{\Sigma_{m}\left(m+E-V_{1}\right)}, \sqrt{1-\left(4 V_{2} \Sigma_{m}\right) / q}\right)}(1-2 q s), \\
& f(s)=2 i \alpha \Sigma_{m}^{-1} s^{\delta \sqrt{\Sigma_{m}\left(m+E-V_{1}\right)}}(1-q s)^{\frac{1}{2}\left[1+\sqrt{1-\left(4 V_{2} \Sigma_{m}\right) / q}\right]} \\
& \times\left\{\left[-\delta \sqrt{\Sigma_{m}\left(m+E-V_{1}\right)}+\frac{q}{2}\left[1+\sqrt{1-\left(4 V_{2} \Sigma_{m}\right) / q}\right] \frac{s}{1-q s}\right.\right. \\
& \left.-\frac{\kappa}{l n s}\right] P_{n}^{\left(2 \delta \sqrt{\Sigma_{m}(m+E-V 1)}, \sqrt{1-\left(4 V_{2} \Sigma_{m}\right) / q}\right)}(1-2 q s) \\
& +q\left(n+2 \delta \sqrt{\Sigma_{m}\left(m+E-V_{1}\right)}+\sqrt{1-\left(4 V_{2} \Sigma_{m}\right) / q}+1\right) \\
& \times P_{n-1}^{\left(1+2 \delta \sqrt{\Sigma_{m}\left(m+E-V_{1}\right)}, 1+\sqrt{1-\left(4 V_{2} \Sigma_{m}\right) / q}\right)}(1-2 q s) \text {. }
\end{aligned}
$$

where $\delta^{2}=1 /\left(4 \alpha^{2}\right)$ in the above equations.

\section{CONCLUSION}

We have approximately solved the Dirac equation for the Morse, Hulthén, and q-deformed Rosen-Morse potentials with the exact pseudospin symmetry for arbitrary spin-orbit quantum number $\kappa$. We have found the eigenvalue equation, and corresponding Dirac spinors in terms of Jacobi (or Laguerre) polynomials by using the parametric generalization of the NU-method within the framework of an approximation to the spin-orbit coupling term. We have observed that the parametric form of the NU method can be used to solve the Dirac

equation with the above potentials. Our results for the cases of Morse potential is good agreement with the ones obtained in the literature.

\section{ACKNOWLEDGMENTS}

This research was partially supported by the Scientific and Technical Research Council of Turkey. 
[1] A. Arima, M. Harvey, and K. Shimizu, Phys. Lett. B 30, 517 (1969).

[2] K. T. Hecht, and A. Adler, Nucl. Phys. A 137, 139 (1969).

[3] J. Meng, K. Sugaware-Tanabe, S. Yamaji, and A. Arima, Phys. Rev. C 59, 154 (1999).

[4] J. N. Ginocchio, and D. G. Madland, Phys. Rev. C 57, 1167 (1998).

[5] J. N. Ginocchio, Phys. Rev. Lett. 78(3), 436 (1997).

[6] J. Meng, K. Sugaware-Tanabe, S. Yamaji, P. Ring, and A. Arima, Phys. Rev. C 58, R628 (1998).

[7] R. D. Ratna Raju, J. P. Draayer, and K. T. Hecht, Nucl. Phys. A 202, 433 (1973); J. P. Draayer, and K. J. Weeks, Ann. Phys. (N.Y.) 156, 41 (1984).

[8] T. Beuschel, A. L. Blokhin, and J. P. Draayer, Nucl. Phys. A 619, 119 (1997); A. L. Blokhin, T. Beuschel, J. P. Draayer, and C. Bahri, Nucl. Phys. A 612, 163 (1997).

[9] J. N. Ginocchio, and A. Leviatan, Phys. Lett. B 245, 1 (1998).

[10] A. L. Blokhin, C. Bahri, and J. P. Draayer, Phys. Rev. Lett. 74, 4149 (1995).

[11] C. Bahri, J. P. Draayer, and S. A. Moszkowski, Phys. Rev. Lett. 68, 2133 (1992).

[12] J. N. Ginocchio, Phys. Rep. 414, 165 (2005).

[13] A. Bohr, I. Hamamoto, and B. R. Mottelson, Phys. Scr. 26, 267 (1982); B. Mottelson, Nucl. Phys. A 522, 1 (1991).

[14] D. Troltenier, C. Bahri, and J. P. Draayer, Nucl. Phys. A 586, 53 (1995).

[15] J. Dudek, W. Nazarewicz, Z. Szymanski, and G. Le Ander, Phys. Rev. Lett. 59, 1405 (1987).

[16] F. S. Stephens et al., Phys. Rev. Lett. 64, 2623 (1990).

[17] C. Berkdemir, Nucl. Phys. A 770, 32 (2006).

[18] W. C. Qiang, R. S. Zhou, and Y. Gao, J. Phys. A: Math. Theor. 40, 1677 (2007).

[19] O. Bayrak, and I. Boztosun, J. Phys. A: Math. Theor. 40, 11119 (2007).

[20] A. Alhaidari, Phys. Rev. Lett. 87, 210405 (2001).

[21] C. S. Jia, P. Guo, Y. F. Diao, L. Z. Yi, and X. J. Xie, Eur. Phys. J. A 34, 41 (2007).

[22] Y. Xu, S. He, and C. S. Jia, J. Phys. A 41, 255302 (2008).

[23] A. Alhaidari, J. Phys. A 34, 9287 (2001).

[24] J. Y. Gou, and Z. Q. Sheng, Phys. Lett. A 338, 90 (2005).

[25] C. S. Jia, P. Guo, and X. L. Pery, J. Phys. A: Math. Gen. 39, 7737 (2006). 
[26] L. H. Zhang, X. P. Li, and C. S. Jia, Phys. Lett. A 372, 2201 (2008).

[27] A. Soylu, O. Bayrak, and I. Boztosun, J. Phys. A: Math. Theor. 41, 065308 (2008).

[28] A. Alhaidari, J. Phys. A 37, 5805 (2004).

[29] R. Lisboa, M. Malheiro, A. S. De Castro, P. Alberto, and M. Fiolhais, Phys. Rev. C 69, $024319(2004)$.

[30] A. S. De Castro, P. Alberto, R. Lisboa, and M. Malheiro, Phys. Rev. C 73, 054309 (2006).

[31] C. S. Jia, J. Y. Liu, L. He, and L. T. Sun, Phys. Scr. 75, 388 (2007).

[32] C. Berkdemir, and R. Sever, J. Phys. A: Math. Gen. 41, 045302 (2008).

[33] W. Greiner, B. Müller, and J. Rafelski, Quantum Electrodynamics of Strong Fields (SpringerVerlag, New Yok, 1985).

[34] A. Leviatan, and J. N. Ginocchio, Phys. Lett. B 518, 214 (2001).

[35] C. L. Pekeris, Phys. Rev. 45, 98 (1934).

[36] A. F. Nikiforov, and V. B. Uvarov, Special Functions of Mathematical Physics (Birkhauser, Basel, 1988).

[37] P. M. Morse, Phys. Rev. 34, 57 (1929).

[38] L. Hulthén, Ark. Mat. Astron. Fys. A 28, 5 (1942).

[39] C.-Y. Chen, D.-S. Sun, and F.-L. Lu, Phys. Lett. A 370, 219 (2007).

[40] C. Tezcan, and R. Sever, J. Math. Chem. 42, 387, (2006); C. S. Jia, Y. Sun, and Y. Li, Phys. Lett. A 331, 115 (2003). 